\title{
La gestión de la innovación en las agencias de viajes cubanas
}

DOI: https://doi.org/10.33262/ap.v3i1.37

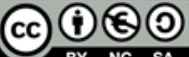

The management of innovation in Cuban travel agencies

\author{
Rebeca Olivera Elosegui ${ }^{1}$ \& Maité Rodríguez González ${ }^{2}$
}

\begin{abstract}
.
Innovation has become one of the fundamental instruments within companies that want to remain competitive in an increasingly complex and changing environment. However, many entities in the Cuban tourism sector, specifically travel agencies, have insufficient Innovation Management and their current situation is unknown. Therefore, the objective of this research is: Assess the management of innovation in general travel agencies, belonging to (OSDE) Viajes Cuba. For this, it was determined to apply in three travel agencies, one of these models of diagnosis and improvement of innovation management: Guide for the Selfevaluation of Business Innovation Management prepared by the Andalusian Center for Excellence in Management, ( 2009), The Model of the 7 Dimensions (7 D's) by Arzola \& Mejías, (2007), and the merger of the model of the Center for Innovation and Business Development of the Generalitat de Catalunya (CIDEM) developed in 2002 and the procedure for the evaluation of Innovation Management proposed by the same institution in 2009. The stages corresponding to the diagnosis within these methodologies included the application of questionnaires, interviews with managers, in-depth interviews with $\mathrm{R}+\mathrm{D}+\mathrm{i}$ specialists and documentary analysis. The diagnosis and application of the Vester Matrix, the problem tree and the objectives tree, made it possible to identify the existing gaps between the ideal and
\end{abstract}

\footnotetext{
${ }^{1}$ Universidad de La Habana, Facultad de Turismo. La Habana, Cuba. rolivera@ftur.uh.cu

${ }^{2}$ Universidad de La Habana, Facultad de Turismo. La Habana, Cuba. mrodriguez@ftur.uh.cu
} 
current state, allowing to structure the logical framework for improvement, as well as to establish a set of indicators for planning and control in these tourist institutions..

Keywords: innovation management, travel agency, diagnosis, actions.

\section{Resumen.}

La innovación se ha convertido en uno de los instrumentos fundamentales dentro de las empresas que quieren seguir siendo competitivas en un entorno cada vez más complejo y cambiante. No obstante, muchas entidades del sector turístico cubano, específicamente las agencias de viajes, presentan una insuficiente Gestión de la Innovación y se desconoce cuál es su situación actual. Por lo que se plantea como objetivo de la presente investigación: Valorar la gestión de la innovación en las agencias de viaje generalistas, pertenecientes a (OSDE) Viajes Cuba. Para ello se determinó aplicar en tres agencias de viajes, uno de estos modelos de diagnóstico y mejora de la gestión de la innovación: Guía para la Autoevaluación de la Gestión de la Innovación) Empresarial elaborada por Centro Andaluz para la Excelencia en la Gestión, (2009), El Modelo de las 7 Dimensiones (7 D's) de Arzola \& Mejías, (2007), y la fusión del modelo del Centro de Innovación y Desarrollo Empresarial de la Generalitat de Catalunya (CIDEM) desarrollado en el año 2002 y el procedimiento para la evaluación de la Gestión de la Innovación propuesto por la misma institución en el 2009. Las etapas correspondientes al diagnóstico dentro de estas metodologías incluyeron la aplicación de cuestionarios, entrevistas a directivos, entrevista a profundidad a especialistas de $\mathrm{I}+\mathrm{D}+\mathrm{i}$ y análisis documental. El diagnóstico y la aplicación de la Matriz de Vester, el árbol de problemas y el árbol de objetivos, posibilitaron la identificación de las brechas existentes entre el estado ideal y actual, permitiendo estructurar el marco lógico para la mejora, así como establecer un conjunto de indicadores para la planificación y el control en estas instituciones turísticas.

Palabras claves: gestión de la innovación, agencia de viajes, diagnóstico, acciones.

\section{Introducción.}

La innovación se ha convertido en el entorno cubano actual, no solo en un término de primer orden, sino un imperativo, para que ese pensamiento "país", se convierta en un pensamiento de "país competitivo", toda vez que están claramente establecidos por múltiples autores los nexos entre innovación, competitividad y ventajas de esta índole.

Aludir a la innovación, es prácticamente un resultado. Shumpeter (1947) planteaba de forma resumida que esta es hacer cosas nuevas o bien el hacer cosas que ya se están haciendo pero de forma distinta, por lo tanto, es la acción de hacer; sin embargo en la consideración de que la empresa es un sistema sociotécnico, no puede pretenderse el resultado sin un proceso para su alcance, y este propio proceso no es espontáneo, sino que con la consideración de los múltiples factores incidentes, debe estar diseñado y armónicamente integrado para el 
cumplimiento de los objetivos; aparece entonces el término de gestión. Ratifican este enfoque López \& Odriozola (2009) al plantear que "La innovación constituye una capacidad organizativa que hay que desarrollar. Como consecuencia, la innovación debe ser abordada como una disciplina de gestión, por cuanto constituye un proceso sistemático, aunque se trate de trabajar con ideas y con la creatividad".

De tal forma este proceso, quizá con visión y enfoques diferentes pero no divergentes, comprende la organización y dirección de los recursos humanos, materiales y económicos en aras del logro de generación de valor, independientemente de la clasificación que adopte la innovación - entre otras, producto, proceso, comercialización y organización (OCDE, 2005), y por tanto debe ser gestionado de manera rigurosa y eficiente (Confederación de Empresarios de Málaga, 2001), toda vez que incorpora los componentes y sus interacciones.

La innovación debe ser considerada un recurso en los procesos de producción de bienes y servicios y convertirse en un medio para alcanzar metas organizacionales. Esta imbricación de la innovación en la empresa, exige de la estructura, cultura y estilos de dirección, características específicas que tributen al éxito de la misma, que han sido definidas como leyes, extraídas de la experiencia práctica de empresas de éxito por Bonet, (2012) quien plantea el apoyo del ápice directivo, el fomento del compromiso, los recursos (presupuestación, disponibilidad, asignación y control), la captación de ideas innovadoras de todas las fuentes, el desarrollo del trabajo en equipo y la visión a futuro.

De tal forma, la gestión de la innovación para Blanco, Bertieri Quintero, \& Villamarín Marentes (2010) está en función de cuatro procesos empresariales que representan cómo un negocio típico puede mejorar su rendimiento: estrategia tecnológica, adquisición de tecnología, desarrollo de nuevos productos e innovación de procesos, los cuales deben estar integrados y ser coherentes con la infraestructura del negocio y sus organizaciones asociadas, por la gestión de personal, los sistemas de control financiero, los asuntos legales, la gestión de calidad y medioambiental.

Si por una parte, múltiples definiciones de gestión de la innovación están signadas por la orientación tecnológica, o de producción material, por otra, de forma general resultan en gran parte válidas para la actividad de los servicios.

Las características de simultaneidad, intangibilidad, importancia de los recursos humanos y la información de los servicios, determinan en cuanto a innovación, la fragilidad de la frontera tipológica entre innovación de producto y proceso, el rol fundamental de las tecnologías de la información y la comunicación, la incidencia de los factores organizativos y -al menos teóricamente- la mayor orientación hacia la adaptación a las necesidades de los clientes (Sirilli y Evangelista, 1998; Torres y Jacob, 2001 y Hipp et al, 2000 citados por Jacob y Aguiló, 2008). 
Múltiples valoraciones indican la relación más íntima de la gestión de la innovación en los servicios con la calidad y la excelencia (Arzola \& Mejías, 2007), el factor humano (Den Hertog \& Bilderbeek, 1999), y la innovación abierta (Vargas, 2013), dada la potencialidad de proveedores y prestatarios para la generación de ideas y el propio cliente, a partir de su carácter como elemento base del propio servicio. (Eiglier \& Langeaurd, 1989), aun cuando, estudios financiados por la Fundación COTEC para la innovación tecnológica, identifican barreras para la innovación en los servicios similares a las existentes en la actividad manufacturera.

Entre ellas las legales y burocráticas, restricciones financieras, (el factor costos ha sido identificado como el de mayor incidencia en las empresas de servicios latinoamericanas por Crespi \& Olivari en 2016), los problemas técnicos donde los más comunes que impiden la introducción o la difusión de innovaciones en servicios suelen ser el escaso conocimiento tecnológico de los políticos que deciden y conceden proyectos y ayudas de I+D y la no disponibilidad de las máquinas (tecnología) adecuadas para los propósitos de pequeñas empresas, capacidades de absorción de los mercados (más limitadas en los servicios), falta de experiencia en gestión de innovaciones, déficits de conocimientos y falta de cultura organizativa de la innovación(Jacob, Tintoré, \& Torres, 2001),

En el orden práctico, se requeriría de un adecuado y contemporáneo enfoque de visión hacia adentro y hacia afuera de la empresa, a corto y mediano plazos, que necesariamente no desarrollan todas las empresas.

Con relación al mercado, Antón \& Duro, (2009), en particular para la actividad turística, visualizan en el mismo los tres factores que la compulsan: la demanda, la oferta y la competencia.

Es de considerar en el sector, lo expuesto por Alzua \& Abad, (2008), quienes plantean que la tendencia de la dinámica de la innovación en el mismo es incremental, al corresponderse la incorporación de nuevos atributos o la mejora de los existentes, en respuesta al comportamiento del mercado y al interés por ganar cuota en el mismo.

Sin embargo, resulta evidente, que el turismo en su conjunto, se ha desarrollado como un sector altamente innovador. Con la clasificación de producto de Martín, (2010) han aparecido nuevos destinos, nuevas instalaciones y nuevos viajes; a su vez estos han introducido nuevos conceptos. Se habla hoy de destinos inteligentes, hoteles parque y experiencias, por ejemplo. Por otra parte, en la actividad innovadora del sector inciden las tendencias medioambientales, el desarrollo energético, la electrónica, por solo mencionar algunos; mientras que las tecnologías de la información y las comunicaciones, no solo han revolucionado la comunicación promocional, las reservas, las ventas y la distribución en general; sino que han invadido los procesos back office, en búsqueda de una mayor fiabilidad y menor tiempo de respuesta, exigencia del cliente turístico del siglo XXI. "La innovación en el sector turístico 
es una combinación de dos factores: la investigación aplicada en él (producción de conocimientos), y la capacidad de las empresas para absorber nuevas tecnologías y conocimientos (distribución de conocimientos), la cual depende de su capacidad para absorber dichos conocimientos, y de la capacidad que tienen las organizaciones de cambiar para transmitirlos de forma eficiente. Y sin duda, ésta es la pieza clave de la competitividad en el sector turístico" (Sancho \& Llaudes, 1999).

Todo este amplio espectro constituye el campo del juego innovador de las agencias de viaje, las cuales por una parten "importan" en sus productos integrados la innovación de sus prestatarios, diseñan y rediseñan productos viajes, mientras por otra, acuden a nuevos modelos de negocios, sistemas de gestión y competencias organizacionales.

Algunas investigaciones en las últimas décadas, en cuanto a la innovación en las agencias de viajes, presentan que:

- Los aspectos en los que las agencias consideran que deben invertir de cara al futuro son, en principio, la forma de atraer nuevos clientes, el modo en que se gestionan los presupuestos y las reservas, el acceso a la información de proveedores y destinos, la manera de comunicarse con los viajeros. (Agencia Europa Press, 2019)

- Las agencias de viajes más grandes (de más de 250 empleados), son las que mayor número de innovaciones introducen. (Jacob, Tintoré, Simonet, \& Aguiló, 2004)

- Los apartados objeto de inversión en cuanto a innovación en las agencias de viajes, son, en orden descendente: producto, comercialización y procesos. (Amadeus, 2016)

- La tecnología clave es la informática y las nuevas tecnologías de la información. (Alfonso, Rodríguez, \& Blanco, 2012)

- La innovación en las agencias se centra en los sistemas centrales de reservas (CRS), Globales de distribución (GDS), los gestores de viajes automáticos o el comercio on line de los productos turísticos (Esteban \& Rubio, 2013)

Si se valora además, que el turista actual es cada vez más independiente, demanda más productos a la medida y en ocasiones se requiere de un diseño personal e individualizado (Esteban \& Rubio, 2013), más servicios y mayor calidad (Alberdi \& Rubio Andrada, s/f), entonces, la innovación constituye un reto e impacta en el desempeño de las tres funciones básicas de las agencias de viaje: asesoría, producción y distribución, exigiendo su planeación, ordenación y control a través de la gestión.

Es válido destacar que la innovación como tal, o sea la puesta en valor de una idea, es el resultante de todo el proceso. La innovación a partir de la espontaneidad o de la urgencia, se enfrenta a un mayor rango de incertidumbre, si bien puede tener éxito.

"El factor innovador ha pasado a constituir un vector estratégico que permite que la empresa mejore su posición competitiva. Es necesario, por tanto, gestionar los denominados procesos de innovación para que las organizaciones adquieran mayor capacidad de adaptación y, sobre 
todo, la posibilidad de anticipar, e incluso, provocar rupturas que las faculten para renovar sus ventajas competitivas en el momento oportuno". (Hidalgo, 2011). Este mismo autor valora la gestión de la innovación, no solo puertas afuera de la empresa, sino como instrumento para el desarrollo y consolidación de las relaciones internas entre departamentos e individuos de la organización, al fomentar su cultura y sus valores compartidos. (Hidalgo, 2011)

En consideración con el carácter de los servicios, inherente así mismo a la actividad de las agencias de viajes, la gestión de la innovación según Schilling \& Werr , (2009) debe considerar como aspectos clave la gestión del conocimiento para la innovación, la gestión de una red de relaciones, la formalización del proceso de innovación, la participación cruzada y multidisciplinariedad, el involucramiento de los clientes, la incorporación de los empleados, clima organizacional propicio, la comunicación con todos los actores de la cadena productiva y la política interna.

En el contexto empresarial cubano, los resultados, la innovación en sí resulta débil en múltiples sectores, aun con el imperativo económico, la voluntad política para ello, y el marco regulatorio del Decreto 281, que la establece como un subsistema. De hecho este sistema empresarial en Cuba, se encuentra sometido a un proceso innovador de gestión, "que procura impactar en las condiciones de vida de la sociedad, con empresas que logren sostenidamente mayor eficiencia y aportes a la sociedad"

Una aproximación a esta problemática en la actividad turística de la capital cubana, indica que existe reducido número de actividades de investigación, desarrollo y estudio de temas relacionados con la actividad de la innovación turística, no existe actividades de vigilancia del entorno y sistematización de la información sobre competencia, clientes y grupos de interés, una baja cultura de tolerancia al error, lo que limita el cambio y los procesos de creatividad de las empresas (Gamboa, 2015), un deficiente sistema de inteligencia empresarial, no se desarrolla la auditoria de capacidades, la capacitación está enfocada a lo profesional y a la asimilación de las tecnologías de Información y comunicaciones (TIC) y la calidad no se utiliza como un elemento estratégico dentro de la empresa (Álvarez, 2017), innovación intuitiva, déficit de conocimiento y capacitación acerca de la innovación y gestión en correspondencia con las características propias del sector, por parte de sus trabajadores y en especial de los directivos de empresas. (Olivera \& García, 2017)

Estos estudios, no segmentan sus resultados por tipo de negocio turístico; razón que sustenta la necesidad de abordarlo en cada uno de ello, dada la coherencia que con la naturaleza de la empresa debe tener la gestión de la innovación. Se relaciona con esta idea el planteamiento de Betz (1998) quien plantea que la interacción constante de los diversos sistemas en el proceso de innovación complejiza su gestión y la necesidad de combinar el análisis organizacional, la planificación, la predicción y el marketing entre otros elementes, para su éxito. De tal forma, se infiere, que las empresas en un mismo contexto, en un mismo negocio, 
con productos similares y orientadas a iguales mercados y segmentos, requerirán de pautas muy similares para una adecuada gestión de la innovación, aún cuando las características individuales de cada una le posibiliten o no, la propia innovación.

Específicamente entonces, no se conoce cuál es la situación de la gestión de la innovación en las agencias de viajes cubanas, de las cuales el elemento observable es el tema producto, que se presenta como debilidad en los análisis de las mismas, siendo este solo una de las tipologías de la innovación, según el Manual de Oslo. (OCDE, 2005). Los cuestionamientos serían ¿Cómo hacerlas? ¿Cómo la estamos haciendo? ¿Dónde radican nuestras insuficiencias o deficiencias? ¿Cómo superarlas?

Se define, así como objetivo del presente documento: Valorar la gestión de la innovación en las agencias de viaje generalistas, pertenecientes a (OSDE) Viajes Cuba.

El cumplimento de este objetivo, a partir de la identificación de las brechas existentes entre el estado ideal y actual, permitirá estructurar el marco lógico para la mejora, así como establecer un conjunto de indicadores para la planificación y el control en estas instituciones turísticas.

\section{Métodos}

Para el cumplimiento del objetivo planteado, se restringe el universo de las agencias de viajes cubanas, a aquellas generalistas pertenecientes a la Organización Superior de Dirección Empresarial (OSDE) Viajes Cuba, se sustenta por una parte en agrupar estas en su conjunto la mayor cantidad de operaciones de esta naturaleza, en el país. Por otra parte, es necesario observar que las agencias de viajes especializadas en producto mercado, dada las diferencias en este pueden abordar los procesos de forma más heterogénea toda vez que tal y como se expuso anteriormente en este trabajo la gestión de la innovación debe ser coherente con los componentes organizacionales, operativos y comerciales de las instituciones. Fundamentan además esta discriminación, elementos objetivos tales como acceso a la información, mayor tiempo en operación de las agencias e interés expreso de la OSDE en la investigación.

Se realizó la investigación en las agencias Cubatur S.A., Havanatur S.A. y Cubanacán S.A.

Una aproximación a la gestión de la innovación, requeriría el fundamento conceptual de los modelos para ello; sin embargo, si bien estos posibilitan la innovación sistémica a través de la comunicación, estandarización y la mejora, en la actualidad - tras el tránsito de la primera a la quinta generación - muchos de ellos, por el propio devenir temporal, se adecuan de forma limitada a la actividad de servicios.

Si bien, cada una de estas generaciones ha producido aportes significativos, algunos investigadores concluyen que no existe un modelo único o generalizable y King \& Anderson, (2003) plantean que la mayoría de estos modelos han resultado ser incapaces de capturar toda 
la complejidad de la realidad de este proceso y actualmente coexisten en sus diferentes formas.

Mientras Salazar, Arzola, \& Pérez, (2010) defienden la precisión de un modelo de gestión de la innovación, Güell, (2014) sustenta la utilidad de los mismos "en la medida que facilitan la explicación de una realidad compleja y la transmisión de experiencias de otros casos, así como la implementación de procesos, no porque

Las autoras consideran, orientadas a la valoración de la gestión de la innovación y por tanto, la utilización de un diagnóstico como herramienta, que los modelos resultan fundamento y punto de partida para tal propósito. Sin embargo para la actividad investigativa, resulta necesaria la identificación previa de pautas para la selección de aquellos a utilizar. Se determinaron como tales:

- Integración en contenido del modelo y del diagnóstico.

- Presentación explícita de pautas para la elaboración de instrumentos para la captación de información, o incorporación de los mismos.

- Adecuación a los servicios y por tanto a las agencias de viajes.

- Posibilidad de adaptación o disruptiva, a partir de los objetivos de la investigación.

Se seleccionaron tres modelos de diagnóstico y mejora de la gestión de la innovación, cada uno ser aplicado en una de las agencias de viajes seleccionadas, de forma aleatoria, con el fin metodológico de obtener una mayor fiabilidad en los resultados generales, independientemente del modelo y métodos empíricos aplicados en cada entidad.

Con la finalidad del conocimiento de los modelos y sus indicadores a analizar (en lo adelante, generalizados como ítems) se presentan a continuación:

a) El Modelo, cuestionario y buenas prácticas de gestión en innovación empresarial (Guía para la Autoevaluación de la Gestión de la Innovación) Empresarial elaborada por Centro Andaluz para la Excelencia en la Gestión, (2009), que define los siguientes criterios:

- Estrategia y Cultura de Innovación: Adquirir un compromiso mediante la manifestación y comunicación de la importancia de la innovación al resto de la organización, definir objetivos coherentes, asumir riesgos derivados de las actividades de innovación, definir la infraestructura necesaria para realizar actividades de innovación, disponer de recursos materiales y económicos necesarios.

- Gestión de los recursos: Proveer los recursos humanos necesarios para mejorar la gestión de la innovación, motivar e ilusionar al personal e impulsar la participación del mismo, fomentar la creatividad y el desarrollo de la capacidad de trabajo en equipo, planificar y gestionar actividades formativas relacionadas con la innovación para alcanzar las competencias necesarias, evaluar la eficacia 
de las acciones realizadas en relación con los recursos humanos con el objeto de mantener y actualizar los registros apropiados de la educación, formación, habilidades y experiencia de las personas de la organización que realizan y gestionan actividades de $\mathrm{I}+\mathrm{D}$.

- Vigilancia del Entorno: Identificación de necesidades, fuentes y medios de acceso de información, búsqueda, tratamiento y difusión de la información, puesta en valor de la información.

- Análisis Interno: Análisis interno, identificación de problemas y oportunidades para la organización.

- Generación y Selección de ideas: Planificar la generación de nuevas ideas, utilizar herramientas para ello, identificar los recursos disponibles que contribuyan a la generación de ideas innovadoras, fomentar la creatividad y el trabajo en equipo para detectar nuevas ideas que permitan guiar el desarrollo de productos y/o procesos futuros para la organización, identificar y desarrollar criterios de selección de nuevas ideas, valorar los factores que garanticen el éxito de nuevas ideas, identificar aspectos relevantes para cada idea: recursos necesarios, escenarios de evolución, impacto en el mercado, contribución a los objetivos de la organización, factores de riesgo, probabilidades de éxito, costes y beneficios esperados.

- Resultados de la innovación: Nuevos productos, procesos o servicios, incremento de las ventas, reducciones de costes de recursos por producto o servicio, reducciones de tiempo en los procesos de producción, distribución o comercialización, incremento de la productividad, mejora de la calidad de los productos, procesos o servicios existentes, satisfacción del cliente con las actividades de innovación, margen de explotación, publicaciones científicas, artículos en revistas profesionales y técnicas, incorporación de tecnologías en la organización.

No se analizó el criterio 6. Gestión de los proyectos de innovación, a partir de los resultados obtenidos por Álvarez (2017) indica que en las entidades de turismo no se trabaja por proyectos.

b) El Modelo de las 7 Dimensiones (7 D's) de Arzola \& Mejías, (2007), orientado a la gestión de la innovación en los servicios y conceptualizado sobre los criterios de los modelos de excelencia. Las dimensiones son:

- Liderazgo: Ejercicio de autoridad, responsabilidades, motivación, toma de decisiones, mecanismos para fomentar la innovación y políticas.

- Planificación Estratégica: Conocimiento y uso adecuado de las capacidades internas, considerando las oportunidades y amenazas del entorno para formular las estrategias a seguir: la innovación como elemento indispensable en la formulación de la visión, misión, estrategias, mejoramiento continuo, recursos 
invertidos en innovación, activos de propiedad intelectual, inversión en formación de personal, vigilancia, plan de desarrollo tecnológico.

- Competencias del Recurso Humano: Conocimientos, habilidades y formación del personal necesarios para que el servicio ocurra en la calidad y oportunidad esperada por el cliente: formación, capacitación, participación en programas de mejoras e innovación, creatividad, trabajo en equipo, motivación, recompensas, sistema de gestión del RRHH y las fuentes de aprendizaje.

- Procesos: Aspectos asociados con la infraestructura de los procesos necesarios para la prestación del servicio, tanto de naturaleza tangible como intangible: tecnología utilizada, documentación, certificación, mejora continua, tipo, uso de la capacidad.

- Organización: Estructura organizativa, manuales, normas, procedimientos, sistemas de información, seguimiento y control, ambiente de trabajo adecuado y la incorporación de mejoras en estos ítems.

- Satisfacción de Clientes:

- Responsabilidad Social: Exigencias actuales de la sociedad, clientes, accionistas, trabajadores, ambiente y la sociedad en general.

c) La fusión del modelo del Centro de Innovación y Desarrollo Empresarial de la Generalitat de Catalunya (CIDEM) desarrollado en el año 2002 y el procedimiento para la evaluación de la Gestión de la Innovación propuesto por la misma institución en el 2009, a partir de que el primero no presenta de forma explícita los aspectos vinculados a la organización, destacados anteriormente en el presente trabajo como de importancia. Se redefinen como pilares así:

- Compromiso de la dirección y liderazgo.

- Cultura de la innovación: Importancia prestada a la gestión de la innovación, reconocimiento de la necesidad de la gestión de la innovación, incorporación de la innovación en la comunicación interna y externa de la dirección, conversión de las competencias individuales en organizacionales, disposición ante el riesgo.

- Generación de nuevos conceptos: Identificación de necesidades actuales y futuras, estimulación de la creatividad de los trabajadores, planificación de la generación de nuevos conceptos, filtrado y selección de ideas, aplicación de herramientas para la generación de nuevos conceptos.

- Desarrollo de Productos: Gestión de la información de partida para un nuevo producto, participación de las áreas de la empresa, clientes y proveedores, planificación temporal por fases y costos previstos, grado de incorporación de nuevas tecnologías, empleo de herramientas para el desarrollo de productos:

- Redefinición de procesos de producción: seguimiento de tecnologías y modelos de organización de los procesos productivos, planificación de recursos financieros, estrategias de subcontratación, oportunidad de innovadora por la 
integración de actividades logísticas, uso de herramientas para la definición y control de procesos.

- Redefinición de procesos de comercialización: Generación de ideas para el proceso de comercialización, planificación para la comercialización de un nuevo producto, redefinición de procesos de comercialización y de marketing con relación al entorno, comunicación postventa, posibilidad de comercialización a través de nuevas tecnologías.

- Gestión del conocimiento y la tecnología: Incorporación de tecnologías clave para la empresa, incorporación de tecnologías al desarrollo de productos, definición de labores internas y necesidad de subcontratación, gestión adecuada del conocimiento, gestión de activos de propiedad intelectual ${ }^{3}$.

Una lectura detallada de los contenidos anteriores posibilita resumir, que los modelos seleccionados recogen para el diagnóstico de la innovación, similares indicadores agrupados en ítems de disímil nomenclatura.

La información requerida fue obtenida a través de la revisión documental, (Balances, Ejercicio Estratégico, Mapa de Procesos), entrevistas a directivos y cuestionarios a trabajadores a partir del cálculo de una muestra probabilística estratificada con un margen de confianza de 95\%, elaborados sobre la base de los contenidos expuestos, así como integrando elementos de Cuestionario de Autoevaluación para valorar las competencias de innovación para pequeñas y medianas empresas, propuesto por la Dirección de Operaciones e Innovación de la ESADE Business School, de España (ESADE, 2005) y Guía de evaluación integrada de la innovación para organizaciones, (Delgado, 2017), procesados por el SPSS.

Aun con la utilización de este tipo de muestra y el análisis cuantitativo posible para el análisis por agencia, se realizó una valoración cualitativa de toda la información obtenida, en observancia al objetivo definido. Tal y como se aprecia en los ítems contentivos en cada modelo seleccionado, a excepción del correspondiente al CIDEM que explicita la Cultura de la Innovación, la evaluación de los restantes siempre tributa a la misma, toda vez que esta está inserta en la propia cultura de la organización.

A partir de los resultados obtenidos, se identificaron las principales causas y efectos del estado de la gestión de la innovación, propiciando -con la consideración de la valoración negativa de la misma- la construcción del árbol de problemas, como una herramienta efectiva para el posterior diseño de objetivos y acciones.

\section{Resultados.}

A partir de la triangulación de la información obtenida, se aprecia que:

\footnotetext{
${ }^{3}$ No considerada en el estudio.
} 
La relación innovación y planificación estratégica no se visualiza en la formulación de la Misión de ninguna de las tres agencias estudiadas, acorde lo planteado por Arzola y Mejías (2007). La evaluación de los ítems Estrategia y Cultura de la innovación (criterio); Liderazgo y Planificación Estratégica (dimensiones); Compromiso de la dirección y liderazgo y cultura de la innovación (pilares), según los modelos aplicados.

Aunque la Visión de dos de las agencias incorpora resultados del proceso innovador como expansión de marca, incremento de cartera de clientes y extensión de mercados, no se encuentra la misma integrada adecuadamente a los componentes de la propia planificación (diagnóstico, objetivos, opciones, acciones).

Los retos y oportunidades para la innovación son débilmente conocidos, en coherencia con un análisis interno de similar valoración.

La estructura, procedimientos y estilos de dirección y la rigidez organizativa es uno de los aspectos, que frena la innovación, susceptible de ser modificado.

Este resultado es coherente con el obtenido por Peñalver, (2020), que identifica en la OSDE rasgos de una organización mecanicista, que emanan hacia sus empresas, al obtener respuestas mayoritarias acerca de la comunicación vertical descendente, autonomía de los profesionales y flexibilidad medio - baja, especialización individual y funcional, y orientación a la estandarización y el control.

Relacionado con ello, aparecen además como factores que obstaculizan la innovación, la intolerancia a los errores, la baja disposición para asumir riesgos y resistencia al cambio.

Inciden además factores externos identificados por Díaz, (2018), tales como el alto grado de centralización, la dificultad de financiación -que se reflejan en insuficientes recursos materiales y equipamiento en las agencias, derivado en alto grado del bloqueo económico financiero impuesto por Estados Unidos al país - y los incentivos insuficientes que inciden en el comportamiento del personal, respecto a la innovación.

No existen fundamentos para definir en qué grado es este último un factor determinante, cuando aparecen también aspectos tanto vinculados a métodos y estilos de dirección, anteriormente planteados, como a la existencia, formación y desarrollo de competencias de los recursos humanos. En cuanto al primero de estos aspectos, los métodos para motivar al personal han sido poco efectivos, no se involucra para la búsqueda de soluciones al colectivo de trabajadores, mientras que la comunicación entre áreas no está formalizada para estos fines; entre otras condicionantes porque la concepción de la actividad innovadora se centra en producto y en las áreas comerciales. No se trabaja en equipo, no se estimula la creatividad de los trabajadores, no existen espacios de intercambio ni mecanismos efectivos para la generación y selección de ideas, al menos con la intensidad que la actual situación competitiva externa (como destino) demandaría e interna (las agencias del país) que resulta 
en una oferta poco diferenciada para todas, a pesar de que la Visión de las tres agencias se orienta al liderazgo en calidad y excelencia.

De forma general, no se pone en valor, la experiencia y el conocimiento de los guías de turismo y su potencial rol como canal de la voz del cliente, a partir de su dominio idiomático y su tiempo de convivencia, que le posibilite obtener de fuente viva, criterio y sugerencias que no necesariamente se reflejan en cuestionarios de satisfacción, quejas o reclamaciones.

Estos elementos fundamentan, la alta dispersión (desviación estándar) entre estratos y al interior de los mismos en las respuestas de los cuestionarios, tanto en cuanto en todos los ítems.

A pesar de la jerarquización y prácticamente unicidad (en cuanto a reconocimiento) de tipología de innovación de producto, no se encuentran estructurados los procesos para su diseño y lanzamiento, la información de mercados y segmentos y la retroalimentación de los clientes se considera insuficiente, mientras que la vigilancia tecnológica se limita a la Informática por lo que se reconoce el débil conocimiento del mercado y de la tecnología como factor que frena la innovación a nivel macro o meta.

La restricción de la innovación en las agencias de viajes a producto, discrimina las funciones de asesoría y distribución de estas empresas, en un contexto actual en el que, por una parte, el cliente es más independiente en su conformación de viaje y por otra, la construcción de su experiencia incluye su relación con la agencia en todas las fases del viaje (antes, durante y después).

Paradójicamente se reconoce la importancia y resultados de la implementación del comercio electrónico, pero no se valora como innovación de proceso y mercadotecnia.

Contradictoriamente con criterios autorales que centran los recursos humanos en la innovación en los servicios, en las tres agencias estudiadas, a través de los tres modelos utilizados, estos en sentido general son considerados como competentes, se destacan en los postulados de Misión, y se percibe, no obstante, un bajo dominio del concepto de innovación y su amplitud y prácticamente un desconocimiento de la gestión de la misma y sus modelos. No es de extrañar entonces, que no tengan definidos modelos de gestión de la innovación.

Se desarrollan planes de capacitación acorde a perfiles profesionales, pero no incluyen acciones relacionadas con la innovación. El trabajo desarrollado por Olivera \& García, (2017) propuso el incremento de la oferta académica en este sentido, cuando en la actualidad se desarrollan en la Universidad de La Habana y La Universidad Tecnológica José Antonio Echevarría como postgrados, mientras que los Centros de Capacitación adscritos a las delegaciones territoriales del Ministerio y su Escuela Ramal, estructuran su oferta docente a partir de la demanda expresa, características y posibilidades de las entidades turísticas; de lo 
que se infiere que no ha sido identificada plenamente por las agencias la necesidad actual y futura de incrementar el conocimiento en cuanto al tema.

Solo en una de las agencias, está definido el desarrollo y la innovación como macroproceso estratégico, pero visualmente no se identifica la relación que posee con los procesos de apoyo, cuando estos pueden impactar en los costos, preocupación prioritaria.

\section{Reflexiones finales.}

La valoración de la gestión de la innovación no difiere en alto grado entre una y otra de las agencias de viajes estudiadas. De forma general, puede evaluarse de débil, identificándose como causas fundamentales las siguientes:

- No visualización de la actividad en la planificación estratégica, lo que de alguna forma indica la percepción limitada de la importancia y necesidad de su gestión.

- Conocimiento débil y fragmentado del personal en cuanto al tema de innovación y su gestión.

- Insuficiente motivación e implicación de los trabajadores en la identificación de problemas y oportunidades y por tanto en la búsqueda de soluciones creativas.

- Insuficiente información del entorno.

- Inefectiva comunicación interna.

Si bien, no se refleja de forma explícita, los resultados obtenidos cuestionan el grado de competitividad de las agencias de viajes, aun con los múltiples indicadores que existen para su evaluación. Es de destacar, además, que se soslayan con toda intencionalidad de las autoras, los incidentes relacionadas con la disponibilidad de recursos, al considerarlos de carácter externo a la empresa, al estar vinculadas al entorno país; sin embargo, la definición primaria de estrategia está relacionada justamente con la asignación de recursos y si no se contempla en la misma, no se asignarán a la actividad independientemente de cualquier situación financiera.

Por otra parte, existen elementos del ambiente externo como la centralización, que no propician la innovación y su gestión; pero el ambiente interno es determinante y el externo está en proceso de cambio.

La innovación no es una tarea, no puede ser un resultado de un proceso espontáneo, sino de un proceso estructurado con ese fin, aun con la flexibilidad, la urgencia y hasta las crisis que aparecen en la actividad turística y por ende, de las agencias de viajes. La propia actividad de las agencias de viaje en Cuba, posibilita la diversificación de fuentes para la innovación, más allá de las propuestas de diseño de producto, emanadas de trabajos de diploma u otros de la Licenciatura en Turismo (no aplicadas en gran parte, aún con los avales de las entidades), su función de distribuidoras les posibilita incorporar a su desempeño empresarial ideas de turoperadores, agencias partner y competidoras, hoteles, restaurantes, otros 
prestatarios turísticos y no turísticos, de sus especialistas y áreas funcionales, de sus gestores de ventas y de los propios clientes finales, los turistas.

Las causas identificadas, se relacionan con otros sistemas y herramientas de gestión, imprescindibles para la innovación empresarial como Gestión del Conocimiento, Gestión de la Información, Gestión de Capital Humano y Gestión de la Comunicación Organizacional. Resulta en extremo difícil, si no imposible, deslindar o establecer fronteras entre una y la otra en cuanto a la innovación. Aproximarse a un algoritmo para ello, partiría de la información, fuente del propio conocimiento, el cual si no se organiza no se comunica, no se comparte de forma estructurada entre los miembros de la empresa y solo cuando eso sucede el conocimiento individual se convierte en organizacional, mientras que la visión estratégica tributa a la identificación de las necesidades futuras de conocimiento y al esbozo del perfil del personal que la empresa exigirá y sustenta un comportamiento proactivo de la misma.

\section{Conclusiones.}

- La valoración de la gestión de la innovación en las agencias de viajes, deriva y demanda el diagnóstico de la gestión del conocimiento, de mercadotecnia, de capital humano, de la información y de la comunicación.

- La mejora de la gestión de la innovación, implicará además definir objetivos y acciones que propicien, actualizar los ejercicios estratégicos, definir estrategias de innovación y valorizar la dirección participativa.

- Un planteamiento conceptual, concibe a las agencias de viaje como dinamizadoras del destino, pero ello implica innovación. Cada uno de los Lineamientos de la Política Económica y Social cubana referidos a Turismo, implica a las agencias de viaje y a la innovación. De no transitar estos caminos o similares, tendremos más de lo mismo; hasta hoy un insuficiente nivel de innovación, el riesgo de no cumplir con su rol conceptual y por transición, con la responsabilidad social del turismo respecto a la economía cubana.

\section{Referencias bibliográficas.}

Agencia Europa Press. (11 de Febrero de 2019). Las agencias de viajes valoran la innovación en su negocio aunque mantienen su inversión. Obtenido de http://www.europapress.es

Alberdi, C. E., \& Rubio Andrada, L. (s/f). Madrid+d. Obtenido de Madrid+d Web site.

Alfonso, R., Rodríguez, A., \& Blanco, G. (2012). . La gestión tecnológica e innovación en el sector turístico y elementos para la selección de estrategias. .

Alpízar, G. L. (2019). EL financiamiento a la innovación en Cuba: La experiencia del Fondo Finaciero de Ciencia e Innovación. Economía y Desarrollo . 
Álvarez, L. (2017). La gestión estratégica de la innovación en las entidades turísticas del destino La Habana. Trabajo de Diploma en opción al título de Licenciada en Turismo, Facultad de Turismo, Universidad de La Habana.

Alzua, A., \& Abad, M. (2008). Innovación en la actividad turística vasca: Hacia un modelo competitivo en la C.A. de Euskadi. Revista de Ocio y Turismo, 65-86.

Amadeus. (2016). La innovación de las agencias de viaje se sitúa en posición intermedia y supera a otras actividades del sector. Obtenido de http://www.amadeus.com

Antón, S., \& Duro, J. (2009). Competitividad y Sistemas de Innovación Territorial en Turismo. Obtenido de http://www.urv.cat.

Arzola, M., \& Mejías, A. (2007). Modelo conceptual para gestionar la innovación en las empresas del sector servicios. Revista Venezolana de Gerencia [en línea]., 12(37).

Blanco, F. S., Bertieri Quintero, J., \& Villamarín Marentes, J. (Abril de 2010). Innovación, conceptos básicos. Madrid. Obtenido de ReaserchGate Web site: www.reaserchgate.net

Bonet, A. (2012). LAs 7 Leyes inmutables del éxito en la innovaciínn. Obtenido de http://www.angelbonet.com

Castro, J. A., \& Aura Paulina Flores Barrera. (2017). Gestión de la innovación para la sustentabilidad en turismo: una revisión teórica para el sector hotelero. Revista Espacios.

Confederación de Empresarios de Málaga. (2001). Guía Práctica de Innovacion para PYMES. Málaga.

Crespi, \& Olivari. (2016). La política de innovación en América Latina y el Carbe. Nuevos Caminos. Obtenido de https://publications.iadb.org

Delgado, M. (2017). Temas de Gestión empresarial.Volumen II. Innovación empresarial. La Habana: Editorial Universitaria Félix Varela.

Den Hertog, P., \& Bilderbeek, R. (1999). Conceptualising service innovation and service innovation patterns. Research Program on Innovation in Service, Dialogic, Utrecht.

Díaz, I. (Enero - Junio de 2018). La innovación en empresas estatales cubanas: análisis para un debate. Economía y Desarrollo (on line), 159(1).

Eiglier, P., \& Langeaurd, E. (1989). Servucción: El marketing de los servicios. España: Mc Graw Hill.

ESADE. (2005). Guía práctica de la innovación para Pymes [en línea]. Barcelona: Barcelona: Obtenido de http://www.inpyme.es/index.php/concepto/89

Esteban, C., \& Rubio, L. (2013). Agencias de viajes innovadoras. Ventas, asesoramiento y gestión. . 
Fernández, I. D. (2013). Desafíos de la innovación en Cuba. Habana.

Gamboa, G. (2015). Una mirada a la gestión de la innovación en las empresas del Sector Estatal del Turismo en el destino La Habana. Trabajo de Diploma en opción al título de Licenciado en Turismo, Facultad de Turismo, Universidad de La Habana.

Güell, F. (2014). Un modelo de gestión de la innovación. Girona: Universidad de Girona.

Hidalgo, A. (2011). La gestión de la innovación como proceso. Conocimiento, innovación y desarrollo. Universidad de Costa Rica.

Jacob, M., \& Aguiló, E. (2008). La innovación en el sector turístico: el caso de Baleares. ROTUR. Revista de Ocio yTurismo(NO.1), 51-64 pp.

Jacob, M., Tintoré, J., \& Torres, X. (2001). Innovación en servicios. Innovacion en el sector turístico balear. Análisis prospectivo de tecnologías. Madrid: COTEC.

Jacob, M., Tintoré, J., Simonet, R., \& Aguiló, E. (2004). Pautas de innovación en el sector turístico Balear. Madrid: COTEC.

Jiménez, B. (2012). Procedimiento de Evaluación y Mejora de la Gestión de La Tecnología y la Innovación en Hoteles Todo Incluido. Matanzas.

King, N., \& Anderson, N. (2003). Cómo administrar la innovación y el cambio. Guía crítica para organizaciones. Australia: Editorial Thomson.

López, S., \& Odriozola, U. (18 de Enero de 2009). Cómo gestionar la innovación. Una metodología práctica. Obtenido de http://www.researchgate.net

Martín, R. (2010). Principios, Organización y Práctica del Turismo. Primera Parte. La Habana: Editorial Félix Varela.

Martínez, C. I. (20 de enero de 2020). Portafolio. Obtenido de Portafolio Web site.

OCDE. (2005). Manual de Oslo: Guía para la recogida e interpretación de datos sobre innovación. París: Tercera Edición.

Ohme, E. T. (2002). Guía para gestionar la innovación. Barcelona: Activos de Comunicación Visual S.A.

Olivera, R., \& García, Y. (2017). La innovación en el turismo. De la academia a la praxis. Ponencia en el evento GESEMAP. La Habana.

Peñalver, P. (2020). Diseño de un área administrativa funcional para la gestión de la innovación en la OSDE Viajes Cuba. Trabajo en opción al Título de Licenciada en Turismo. Faacultad de Turismo, Universidad de La Haba.

Salazar, M., Arzola, M., \& Pérez, E. (2010). Gestión de la innovación para las Pymis de Ciudad Guayana. Revista Venezolana de Gerencia [en línea]., 15(51). 
Sancho, A., \& Llaudes, A. (1999). Sector turístico e innovación: Un análisis a través de las patentes. Turitec, 15-17.

Sancho, A., \& Maset, A. (2001). Innovación tecnológica y calidad en el sector turismo. Valencia: Springer Computer Science.

Schilling, A., \& Werr, A. (2009). Managing and organizing for innovation in service firms. Stockholm.

SECTUR. (2013). Programa Sectorial de Turismo 2013-2018. Ciudad de México.

Vargas, L. E. (2013). Diseño de una propuesta metodológica para gestionar la innovación en empresas desarrolladoras de software integrantes de la organización Network Clúster TIC del Triángulo del Café. Trabajo de investigación para optar al título de Magister en Administración. Manizales: Universidad Nacional de Colombia. Facultad de administración. 


\section{Para citar el artículo indexado}

Olivera Elosegui, R., \& Rodríguez González, M. (2020). La gestión de la innovación en las agencias de viajes cubanas. AlfaPublicaciones, 3(1), 14-32. https://doi.org/10.33262/ap.v3i1.37

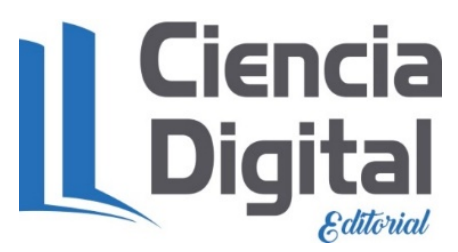

El artículo que se publica es de exclusiva responsabilidad de los autores y no necesariamente reflejan el pensamiento de la Revista Alpha Publicaciones.

El artículo queda en propiedad de la revista y, por tanto, su publicación parcial y/o total en otro medio tiene que ser autorizado por el director de la Revista Alpha Publicaciones.
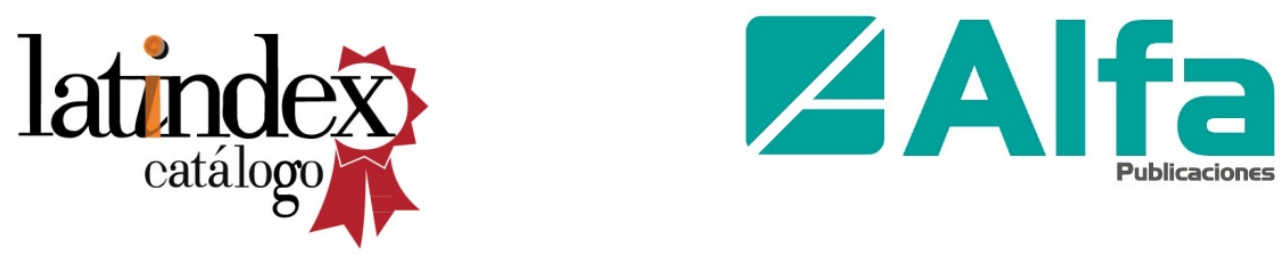